\title{
Cinétiques de transfert des ions phosphate du sol vers la solution du sol : paramètres caractéristiques
}

\author{
JC Fardeau 1, C Morel 1, R Boniface 2 \\ 1 Centre d'études de Cadarache, département de physiologie végétale et écosystème, 13108 Saint-Paul-lez-Durance; \\ 2 CNRA, agronomie, route de Saint-Cyr, 78000 Versailles, France
}

(Reçu le 11 octobre 1990; accepté le 24 août 1991)

\begin{abstract}
Résumé - La nutrition phosphatée des plantes dans un sol peut dépendre de la vitesse de renouvellement des ions phosphate de la solution du sol par des ions de la phase solide. Mais il n'existait pas jusqu'alors de méthode permettant de déterminer ces vitesses de transfert. Grâce à l'emploi de méthodes d'échanges isotopiques dans des systèmes sol-solution en état stationnaire couplées à une analyse stochastique utilisant la méthode des transformées de Laplace, il a été possible de définir : (i) une constante moyenne de vitesse de transfert entre la phase solide et la phase liquide, (ii) un temps moyen de séjour des ions phosphate dans la solution du sol, (iii) un flux moyen de transfert entre la phase solide et la phase liquide. La détermination de ces variables sur des échantillons de terre prélevés dans des parcelles de plusieurs dispositifs expérimentaux de longue durée sur la fertilisation phosphatée a mis en évidence que la constante de vitesse et le temps de séjour sont peu modifiés par les apports d'engrais; par contre le flux l'est fortement par ces derniers. La comparaison des rendements parcellaires et des flux a montré que le seul facteur susceptible de limiter la nutrition phosphatée est bien le flux. L'ensemble de ces résultats conduit à privilégier la présence d'eau dans le sol comme facteur prioritaire pour assurer une nutrition phosphatée satisfaisante même lorsque les stricts besoins en eau sont satisfaits.
\end{abstract}

phosphate assimilable / solution du sol / flux de transfert

Summary - Phosphate ion transfer from soil to soil solution: kinetic parameters. In a soil, phosphate nutrition of crops depends on the rate at which phosphate ions in the soil solution are renewed from phosphate ions in the solid phase. Before the present study, however, no method was available to determine the rate of phosphate ion renewal from soil. Using the isotopic exchange method of ${ }^{32} \mathrm{PO}_{4}$ ions in soil solution systems maintained at steady state and the stochastic analysis of kinetic data (table II), 3 kinetic parameters were defined: (i) the mean renewal rate of phosphate ions from soil which theoretically can vary to a great extent (table IV), (ii) the mean time phosphate ions remain in the soil solution, (iii) the mean exchange flow between soil and solution. These parameters were determined in soil samples (table $V$ ) taken from long-term experimental designs where residual effects of phosphate fertilization were studied. The mean exchange rate and the mean time phosphate ions remain in the soil solution were not greatly modified by crop phosphorus uptake or phosphorus input but the mean exchange flow was greatly modified (table III). Comparison of dry matter yields in the experimental designs with the mean exchange flow shows that the mean flow could be a limiting factor in phosphate nutrition of crops (table III). These results show that attention should be paid to hydric nutrition in order to satisfy phosphorus nutrition.

available P / soil solution / phosphate flow 


\section{INTRODUCTION}

Les plantes cultivées sur des sols où l'agriculture est pratiquée de longue date tirent $80-100 \%$ de leur phosphore dans la réserve assimilable du sol et le complément dans les engrais phosphatés récemment appliqués (Morel, 1988). Ainsi le phosphore du sol assimilable par les plantes et les microrganismes, c'est-à-dire le phosphore susceptible d'être transféré, via la solution du sol, depuis la phase solide du sol vers les végétaux, assure presque à lui seul la satisfaction des besoins des plantes. Ce fait explique que cette forme de phosphore ait fait l'objet de travaux de recherche depuis plus de 100 ans. Dans les méthodes qui font appel à des extractifs chimiques (Daubeny, 1845) le phosphore assimilable d'un sol n'est caractérisé que par un facteur quantité. II peut s'agir «d'une attitude pragmatique utile, mais il serait erroné de la considérer comme suffisante" (Calvet, 1988). Les limites de ces techniques sont apparues avec la multiplication des analyses de terre (Boniface et Trocmé, 1988).

En fait, les chercheurs ont montré, depuis plus de 30 ans (White et Beckett, 1964), qu'une meilleure connaissance du phosphore assimilable du sol nécessite la détermination de 3 paramètres susceptibles de quantifier les facteurs intensité, quantité et capacité (Gachon, 1977). Le paramètre intensité doit illustrer l'activité thermodynamique des ions phosphate dans la solution du sol, phase dans laquelle les plantes prélèvent le phosphore indispensable à leur croissance. Ce facteur est généralement identifié à la concentration des ions phosphate dans la solution du sol (Barber, 1984). Le paramètre quantité doit être représentatif de la quantité d'ions phosphate capables d'alimenter la solution du sol lorsque celle-ci est exploitée par les racines. Le paramètre capacité doit caractériser les variations du facteur intensité lorsque le facteur quantité croît sous l'effet d'apports d'engrais phosphatés ou diminue sous l'effet des prélèvements par les plantes. Bien que constituant un progrès significatif dans la définition et la caractérisation de l'offre alimentaire du sol en phosphore, cette approche restait cependant insuffisante.

En effet, la solution du sol ne contient à un instant donné, dans les cas les plus favorables, que de $2-5 \%$ de la quantité de phosphore prélevé in fine par les plantes. Le phosphore de cette solution du soil doit donc être renouvelé au contact des racines un très grand nombre de fois durant la phase de végétation. Ce fait a parfois été évoqué, soit à l'occasion de considérations théoriques (Calvet, 1988; Triboi et Gachon, 1988), soit à l'occasion de considérations générales lorsque l'offre potentielle du sol en phosphore est définie "comme l'aptitude du sol à fournir un flux d'ions aux surfaces racinaires" (Gachon, 1988). Cependant peu de méthodes ont été proposées pour quantifier le flux potentiel d'ions phosphate entre le sol et la surface racinaire, via la solution du sol. Les tentatives effectuées portent essentiellement sur l'utilisation de résines anioniques dont l'objet est de simuler l'action du prélèvement racinaire (Conesa, 1967; Sibbessen, 1978; Roche, 1983); cependant l'action des résines anioniques sur le sol est très différente de celle des racines notamment par l'ampleur des modifications de $\mathrm{pH}$ qu'elles induisent dans le sol. Aucune méthode expérimentale ne permettait de prévoir le temps nécessaire pour que le facteur intensité retrouve sa valeur initiale lorsque le facteur intensité est modifié par les prélèvements végétaux alors que des méthodes expérimentales ont permis de connaître l'évolution de ce facteur à l'issue d'apports de phosphates (Barrow, 1980; Morel et Fardeau, 1990).

L'objet de cet article est de :

- proposer une méthode permettant de mesurer les flux potentiels d'ions phosphate entre la phase liquide et la phase solide ainsi que celle des flux inverses;

- préciser l'incidence de la fertilisation phosphatée sur les variations de tels flux d'ions phosphate dans le sol en appliquant la méthode à des échantillons de terre issus de divers dispositifs expérimentaux de longue durée sur la fertilisation phosphatée;

- de chercher à savoir si, au contact avec la zone d'absorption racinaire, la vitesse de renouvellement des ions phosphate dans la phase liquide par ceux de la phase solide n'est pas le facteur limitant essentiel de la nutrition phosphatée des plantes dans les sols en place.

\section{MATÉRIEL ET MÉTHODES}

\section{Détermination des cinétiques d'échanges isotopiques des ions phosphate dans les systèmes sol-solution de sol en état stationnaire}

La méthode a été décrite en détail précédemment (Fardeau et Jappé, 1988; Sen Tran et al, 1988). Elle 
consiste, dans son application standard, à agiter un mélange de $10 \mathrm{~g}$ de terre et de $99 \mathrm{ml}$ d'eau désionisée durant une nuit; le système atteint alors un état stationnaire. À l'issue de cette agitation, la concentration des ions passés en solution demeure constante au cours du temps. À un instant $t=0,1 \mathrm{ml}$ d'une solution contenant une quantité $R$ de radioactivité, voisine de $1 \mathrm{MBq}$ et présente sous forme d'ions ${ }^{32} \mathrm{PO}_{4}$, est injecté dans le mélange agité, à 200 tr.min ${ }^{-1}$ environ, par un barreau magnétique couvert de téflon. Sept à $8 \mathrm{ml}$ du mélange sont alors prélevés à l'aide d'une seringue médicale en matière plastique. La solution est immédiatement séparée du sol par passage sur filtres à pores calibrés à $0,2 \mu \mathrm{m}$. Ces prélèvements et filtrations sont effectués à des temps connus qui peuvent être soit $1,10,100,1000 \mathrm{~min}$, soit $1,10,40$ et $100 \mathrm{~min}$ soit encore 1, 3, 7 et 10 min. Les quantités de radioactivité, $r$, restant en solution à l'instant $t$ sont mesurées par comptage en scintillation liquide sur $1 \mathrm{ml}$ des solutions filtrées. À l'occasion du dernier prélèvement, le volume de mélange prélevé est de 15-18 ml; ce mélange est alors filtré sur des membranes à pores de $0,01 \mu \mathrm{m}$ afin de disposer d'un volume suffisant $(10-12$ $\mathrm{ml}$ ) de solution absolument exempte de particules de sol et permettant le dosage du phosphore présent en solution uniquement sous forme d'ions phosphate. La concentration des ions ${ }^{32} \mathrm{PO}_{4}$ est indépendante de la dimension des pores des filtres mais celle des ions $\mathrm{PO}_{4}$ en dépend parfois : certains sols possèdent des particules dispersées d'un diamètre inférieur à $0,2 \mu \mathrm{m}$, qui contiennent du phosphore et qui passent à travers les membranes à pores de 0,2 $\mu \mathrm{m}$ (Fardeau et Jappé, 1988). Ce fait justifie d'utiliser systématiquement, avant tout dosage d'ions phosphate dans la solution du sol, des filtres dont les pores sont calibrés à 0,01 $\mu \mathrm{m}$. Les dosages sont effectués, le plus souvent sur un volume de solution de $9 \mathrm{ml}$, par une méthode céruleo-molybdique avec réduction à froid en présence de tartrate d'antimoine et de potassium (John, 1968).

En faisant appel à cette méthode :

- la concentration $c_{p}$ du phosphore en solution est représentative du facteur intensité;

- la quantité $E_{p i e l}$ de phosphore isotopiquement échangée en 1 min est représentative du facteur quantité (Sen Tran et al, 1988);

- le rapport $E_{\text {pief }} / c_{p}$ est adopté comme facteur capacité. Ce rapport exprime le volume apparent d'ions phosphate présent en phase solide; ce volume apparent est calculé en faisant l'hypothèse que la concentration dans ce volume apparent est égale à celle des ions de la solution.

\section{Principe du calcul des constantes de vitesses et des flux d'échanges des ions phosphate entre la phase liquide et la phase solide du sol}

La méthode des cinétiques d'échange isotopique des ions phosphate pratiquée dans des systèmes en état stationnaire doit aboutir, en toute théorie, à la détermi- nation de 2 types de paramètres permettant de quantifier les transferts d'ions phosphate entre divers compartiments constituant l'ensemble des ions mobiles; le premier type de données doit quantifier les vitesses de transfert des ions entre compartiments et le second type doit quantifier les flux d'ions entre compartiments (Sheppard, 1962).

La démarche expérimentale utilisée permet d'observer directement le devenir de la radioactivité uniquement dans la solution du sol, à savoir le compartiment où la radioactivité a été injectée. L'ensemble des compartiments d'ions phosphate de la phase solide du sol où se distribuent les ions ${ }^{32} \mathrm{PO}_{4}$ lorsqu'ils quittent la solution doit être considéré provisoirement comme une "boîte noire". Dans ces conditions expérimentales l'analyse des caractéristiques cinétiques des mouvements d'ions dans ce système ne peut être entreprise que par la méthode stochastique (Shipley et Clark, 1972). En désignant par $f_{t}$ la fonction représentative de la cinétique de transfert de la radioactivité depuis le compartiment où elle a été injectée à l'instant $t=0$ vers la "boite noire" contenant les ions échangeables, la constante moyenne, $K_{m}$, de vitesse d'échange des ions entre le compartiment d'injection et l'ensemble des autres compartiments est définie par la relation (Shipley et Clark, 1972) :

$$
K_{m}=\int_{0}^{\infty} f_{t} \mathrm{~d}_{t} / \int_{0}^{\infty} t . f_{t} \cdot \mathrm{d}_{t}
$$

Selon l'analyse stochastique, la fonction $\left[f_{t}\right] / R$ est la transformée de Laplace d'une fonction $\mathrm{g}(\mathrm{ki})$ telle que :

$$
\left[f_{t}\right] / R=g\left(k_{i}\right) \cdot \mathrm{e}^{-k_{i} \cdot t} \cdot \mathrm{d} k_{i}
$$

et dans laquelle les $k_{i}$ sont les différentes constantes individuelles de vitesse d'échange entre les ions de la phase solide et ceux de la phase liquide (Sheppard, 1962). La connaissance de la fonction $g\left(k_{i}\right)$, loi de distribution des $k_{i}$, permet de calculer la constante moyenne de vitesse de transfert des ions entre phase solide et phase liquide.

L'inverse de $K_{m}$ est le temps de séjour moyen, $T_{m}$, des ions dans le compartiment où la fonction $f_{t}$ est déterminée. Cette donnée $T_{m}=1 / K_{m}$ représente le temps nécessaire pour qu'une quantité de matière égale à celle présente dans le compartiment d'injection entre et sorte de ce compartiment. Cette définition montre que la valeur numérique de l'inverse de $T_{m}$, c'est-à-dire $K_{m}$, représente également le nombre de fois où, par unité de temps, les ions phosphate de la solution seront renouvelés par ceux originaires de tous les compartiments échangeables de la phase solide.

En désignant par $Q$ la quantité d'élément présente dans le compartiment d'injection, le flux moyen d'échange et de transfert, $F_{m}$, d'ions phosphate entre la phase solide et la phase liquide du sol a pour valeur:

$$
F_{m}=Q . K_{m}
$$




\section{Échantillons de terre}

Les échantillons de terre ont été prélevés dans des parcelles de divers dispositifs expérimentaux de longue durée sur la fertilisation phosphatée mis en place pour étudier les effets à long terme sur les sols et les cultures (essentiellement des céréales) de différentes doses de phosphore apporté sous une forme solubleeau. On rappellera que les prélèvements de terre ont été effectués après la récolte, c'est-à-dire à des périodes suffisamment éloignées de celles des apports pour pouvoir considérer que la transformation dans le sol des phosphates apportés est pratiquement terminée. Les essais choisis, au nombre de 7 , dont les résultats culturaux ont été publiés antérieurement, ont été mis en place soit par l'INRA pour $B \times 21, B \times 23$, $B \times 24, B \times 33$ et $B \times 37$ (Boniface et Trocmé, 1988) soit par l'ITCF pour Herbisse et Tennie (Taureau et Courant, 1988; Taureau et al, 1988); ces essais sont identifiés par les mêmes symboles que ceux utilisés à cet effet dans les publications précédentes. Les caractéristiques physico-chimiques principales des échantillons de terre prélevés au début des expérimentations et quelques précisions sur les essais sont présentées dans le tableau $\mathrm{I}$.

$\mathrm{Au}$ vu et au su des résultats de ces analyses de routine, les échantillons couvrent une large gamme de types de sol et de fertilités phosphoriques. Un accroissement significatif de rendement relativement à l'absence continue d'apport a été observé dans 2 essais (Bx 21 et $B \times 24$ ) dont les sols étaient cependant considérés, selon l'analyse de routine, comme très large- ment pourvus d'après leur teneur initiale ainsi que celle observée après 10 ans de culture sans apport; à l'opposé, aucun accroissement de rendement n'a été enregistré dans l'essai $\mathrm{Bx} 33$ malgré le classement de sol comme pauvre à l'origine (Boniface et Trocmé, 1988). Ces données suffisent à illustrer les difficultés rencontrées pour raisonner la fertilisation phosphatée sur la seule base des résultats des analyses chimiques de terre couramment pratiquées pour caractériser le statut phosphaté du sol.

\section{RÉSULTATS ET DISCUSSIONS}

\section{Expression mathématique des cinétiques de transferts des ions phosphate dans les systèmes sol-solution}

Pour illustrer les relations pouvant caractériser les cinétiques de transfert des ions phosphate dans les systèmes sol-solution, on a présenté, à titre d'exemple, les résultats obtenus sur un des échantillons de terre prélevés à Herbisse en 1981 avant l'implantation de l'essai de longue durée. Les déterminations ont été réalisées ici sur un mélange de $30 \mathrm{~g}$ de terre et $300 \mathrm{ml}$ de solution. La fraction de la radioactivité injectée restant en solution en fonction du temps d'échange isotopique est donnée au tableau II.

Tableau I. Analyses physico-chimiques principales des échantillons de terre et caractéristiques des expérimentations de longue durée.

\begin{tabular}{|c|c|c|c|c|c|c|c|c|c|c|c|}
\hline \multirow{3}{*}{$\begin{array}{l}\begin{array}{l}\text { Nom } \\
\text { des } \\
\text { essais }\end{array} \\
\begin{array}{l}\text { Tennie } \\
72\end{array}\end{array}$} & \multirow{3}{*}{$\begin{array}{l}\begin{array}{l}p H \\
\text { (eau) }\end{array} \\
6,5\end{array}$} & \multicolumn{3}{|c|}{ Granulométrie (\%) } & \multirow{2}{*}{\multicolumn{2}{|c|}{$\begin{array}{c}\mathrm{MO}(\%) \mathrm{CaCO}_{3} \\
(\%)\end{array}$}} & \multirow{2}{*}{\multicolumn{2}{|c|}{$\begin{array}{l}P \text { extrait } \\
\text { mg(P) } \mathrm{kg}^{-1} \\
\text { Dyer } \mathrm{JH}^{-}\end{array}$}} & \multirow{2}{*}{\multicolumn{2}{|c|}{$\begin{array}{l}\text { Bilan Durée } \\
F-E \\
k g(P) h a^{-1}(a n)\end{array}$}} & \multirow{2}{*}{$\begin{array}{l}\text { Différence } \\
\text { sign } \\
\text { rdt }\end{array}$} \\
\hline & & $A$ & $L$ & $S$ & & & & & & & \\
\hline & & 216 & 589 & 195 & 2,44 & 0 & 131 & 105 & 660 & 10 & Non \\
\hline $\begin{array}{l}\text { Herbisse } \\
10\end{array}$ & 7,9 & 250 & 380 & 330 & 3,90 & 650 & 1 & 87 & 580 & 8 & Oui \\
\hline $\begin{array}{l}B \times 21 \\
17500\end{array}$ & 7,9 & 453 & 299 & 248 & 4,0 & 196 & 1 & 175 & 223 & 6 & Oui \\
\hline $\begin{array}{l}\text { Bx } 23 \\
77560\end{array}$ & 7,0 & 378 & 575 & 47 & 3,9 & 0 & 135 & 1 & 658 & 6 & Non \\
\hline $\begin{array}{l}\text { Bx } 24 \\
40400\end{array}$ & 6,3 & 43 & 131 & 826 & 1,4 & 0 & 182 & 1 & 493 & 6 & Oui \\
\hline $\begin{array}{l}B \times 33 \\
11400\end{array}$ & 7,9 & 331 & 452 & 217 & 1,6 & 6 & I & 35 & 368 & 6 & Non \\
\hline $\begin{array}{l}B \times 37 \\
28000\end{array}$ & 7,7 & 273 & 679 & 48 & 1,9 & 4 & 100 & 1 & 603 & 6 & Non \\
\hline
\end{tabular}


Tableau II. Diminution de la fraction de radioactivité, rt $R$, restant en solution en fonction de la durée de l'échange isotopique pour l'échantillon d'Herbisse.

$\begin{array}{lllllll}t(\min ) 1 & 3 & 7 & 10 & 40 & 100 & 1000\end{array}$

$\begin{array}{llllllll}r / R & 0,27 & 0,21 & 0,16 & 0,15 & 0,10 & 0,08 & 0,048\end{array}$

Le calcul de la régression linéaire entre les valeurs de $\log \left(r_{t} / R\right)$ et celles de log $t$ montre que ces données sont très hautement corrélées, $r^{2}=$ 0,99 .

La relation statistique liant les valeurs de $\log \left(r_{t}\right.$ /R) et celles de log $t$ est dons, de la forme générale:

$$
\begin{aligned}
& \log \left(r_{t} / R\right)=\log \left(r_{t} / R\right)-n \log t \\
& \text { soit } r_{t} / R=\left(r_{1} / R\right) \cdot t^{-n}
\end{aligned}
$$

Les résultats observés sur cet échantillon de terre sont en accord avec ceux précédemment publiés (Wiklander, 1950; Larsen, 1967; Fardeau, 1981).

Cette fonction établie empiriquement et statistiquement éprouvée :

- est utilisable dans la majorité des sols (Fardeau et al, 1985) pour des intervalles [1 min-4 mois], la limite de 4 mois étant liée à la période du ${ }^{32} \mathrm{P}$ et non à une éventuelle modification du mécanisme d'échange isotopique;

- résulte de la simplification de la fonction théorique suivante :

$$
r_{t} / R=\left(r_{1} / R\right)\left[t+\left(r_{1} / R\right)^{1 / n}\right]^{-n}+r_{\infty} / R
$$

Les valeurs numériques déduites de la formule théorique (6) sont compatibles avec les faits expérimentaux, même pour les durées d'échange particulières, $t=0$ et $t=\infty$, alors qu'elles ne l'étaient pas avec la formule empirique (5).

\section{Constante moyenne de vitesse d'échange des ions phosphate entre phase solide et phase liquide}

\section{Calcul théorique}

En analyse stochastique, la fonction (6) est la transformée de Laplace d'une fonction $g\left(k_{i}\right)$. La recherche de la fonction $g\left(k_{i}\right)$ est largement facilitée par l'examen de tables de fonctions et de leurs transformées (Sheppard, 1962). L'examen de celles proposées dans la littérature spécialisée la plus récente (Cranck, 1975) fait apparaître que, parmi les fonctions répertoriées, seule une fonction $g\left(k_{i}\right)$, représentative d'une fonction de densité de probabilité $\gamma$ semble pouvoir correspondre à celle recherchée.

$$
g\left(k_{i}\right)=1 / \Gamma n\left(r_{1} / R\right) k_{i}^{n-1} e^{-(r 1 / R))^{1 / n} k_{i}}
$$

La constante moyenne des vitesses d'échange, $K_{m}$, moyenne mathématique des différents $K_{i}$ distribués selon la fonction de probabilité $\gamma$ a pour valeur (Feller, 1966) :

$$
K_{\mathrm{m}}=n /\left[\left(r_{1} / R\right)^{(1 / n)}\right]
$$

\section{Discussion}

La mesure des transferts d'ions $32 \mathrm{PO}_{4}$ de la phase liquide à la phase solide dans les systèmes sol-solution en état stationnaire permet de connaitre avec certitude les transferts existant en sens inverse. Ce fait, qui justifie l'utilisation des cinétiques d'échanges isotopiques réalisées sans addition d'entraîneur pour quantifier les possibilités de passage du phosphore du sol vers la solution, est suffisamment important pour qu'il soit redémontré brièvement ici dans le cas de son application aux ions phosphate échangeables des sols.

Il n'est pas possible d'observer de discriminations isotopiques entre les ions ${ }^{32} \mathrm{PO}_{4}$ et les ions ${ }^{31} \mathrm{PO}_{4}$ dans les mécanismes mis en jeu dans les systèmes sol-solution-plante. Certes, cette discrimination existe certainement mais elle est inférieure à la précision qu'il est possible d'obtenir pour les mesures de quantité de radioactivité. En effet, les variations de teneur isotopique dues à la discrimination isotopique pour un élément tel que $\mathrm{N}$, plus léger que $\mathrm{P}$, ne sont, dans le meilleur des cas, que de l'ordre de 1/1000 (Mariotti, 1982); dans le même temps, la précision sur la mesure d'une quantité de radioactivité ne peut guère être supérieure au $1 \%$ (Coulter, 1969) en raison, entre autres, du caractère aléatoire des désintégrations radioactives; on est donc en droit d'admettre que, dans les systèmes sol-solution-plante, les ions ${ }^{32} \mathrm{PO}_{4}$ et les ions $31 \mathrm{PO}_{4}$ ont le même devenir.

C'est pourquoi, lorsqu'au cours des mesures d'échanges isotopiques entre sol et solution, des ions ${ }^{32} \mathrm{PO}_{4}$ quittent la solution pour gagner la 
phase solide, des ions ${ }^{31} \mathrm{PO}_{4}$ les accompagnent et gagnent aussi la phase solide, le devenir du traceur étant identique au devenir du tracé (Shipley et Clark, 1972). Ce transfert d'ions ${ }^{32} \mathrm{PO}_{4}$ accompagnés d'ions ${ }^{31} \mathrm{PO}_{4}$ s'effectue avec conservation du rapport ${ }^{32} \mathrm{PO}_{4} /{ }^{31} \mathrm{PO}_{4}$ des ions présents dans la solution. Si le transfert d'ions $\mathrm{PO}_{4}$ n'avait lieu que dans le sens liquide $\rightarrow$ solide, la concentration des ions $\mathrm{PO}_{4}$ présents en solution diminuerait au cours du temps; or cette concentration reste constante. Ce résultat expérimental ne peut être expliqué que par le transfert, depuis la phase solide jusqu'à la phase liquide, d'une quantité d'ions ${ }^{31} \mathrm{PO}_{4}$ égale à celle quittant au même instant la solution.

La mesure des flux d'ions ${ }^{32} \mathrm{PO}_{4}$ dans le sens liquide $\rightarrow$ solide renseigne donc bien sur les flux d'ions phosphate dans le sens solide $\longrightarrow$ liquide.

Les flux ainsi déterminés en état stationnaire ne seront représentatifs de ceux du sol étudié que si les mesures sont effectuées sans addition d'entraîneur, ce dernier modifiant bien évidemment, dès qu'il est introduit dans le mélange solsolution, tous les flux d'ions. Cette observation exclut donc tout protocole expérimental faisant intervenir des additions plus ou moins massives d'entraîneur ayant pour objectif de faciliter le dosage du phosphore présent en solution ou extrait par des résines anioniques (Triboi et Gachon, 1988).

\section{Application à la détermination des $\mathrm{K}_{\mathrm{m}}$, $T_{m}$ et $F_{m}$ des ions phosphate d'échantillons de terre différenciés par leur régime de fertilisation phosphatée}

\section{Résultats}

Ils sont regroupés dans le tableau III.

\section{Discussion}

\section{Variations des facteurs intensité,} quantité et capacité

Les variations de $c_{p}$, $E_{p i e l}$ et $E_{P i e l} / c_{p}$ en fonction des différents traitements sont semblables à celles observées antérieurement à l'occasion de l'analyse d'échantillons prélevés dans d'autres essais comportant des protocoles semblables. Les facteurs intensité et quantité croissent avec les quantités de phosphore accumulées alors que le facteur capacité diminue; les variations re- latives de $c_{P}$ et de $E_{P i e l}$ sont supérieures à celles de $E_{P i e l} / c_{P}$. C'est dire que les facteurs intensité et quantité peuvent être modifiés de manière significative par l'homme alors que le facteur capacité apparaît comme une caractéristique forte du sol.

Les résultats les plus originaux concernent les essais de Tennie et d'Herbisse pour lesquels les échantillons prélevés en début d'expérimentation étaient disponibles. Dans ces 2 sols, les valeurs des paramètres caractérisant la fertilité phosphorique ne peuvent être maintenues à leurs valeurs initiales que moyennant des apports de phosphore significativement plus élevés que les quantités exportées avec les récoltes soit respectivement 2 fois et 1,8 fois les exportations. Dans ces 2 sols, le maintien de la fertilité à son niveau initial, acquis d'ailleurs à la suite de fertilisations phosphatées importantes, ne peut être obtenu qu'en maintenant un bilan $F-E>0$. L'analyse des résultats des rendements culturaux montre que, pour le système actuel de production, une telle pratique ne semble pas justifiée dans le cas de l'essai de Tennie : les rendements des cultures de blé et de maïs, dans ce sol profond où l'alimentation en eau n'est pas un facteur limitant, ont été indépendants du niveau de fertilisation, comme le laissaient prévoir les caractéristiques initiales et finales de dilution isotopique (tableau III). Ainsi en 9 ans d'expérimentation, les indices moyens de rendement relativement à $P_{0}\left(8,4\right.$ t.ha $^{-1}$ en maïs et 8,28 t.ha $^{-1}$ en blé) s'établissent pour $P_{1}\left(59 \mathrm{~kg} \cdot \mathrm{ha}^{-1} .2\right.$ ans $^{-1}$ sur le maïs) à 98,6 et 94,3 pour le maïs et le blé respectivement, et pour $P_{2}\left(118 \mathrm{~kg} \cdot \mathrm{ha}^{-1} .2\right.$ ans $\left.^{-1}\right)$ à 99,0 et 99,1 .

\section{Variations des constantes moyennes de vitesse d'échange}

Les variations des $K_{m}$ d'un sol donné (tableau III, colonne 8) en fonction des traitements fertilisants antérieurs sont inférieures aux différences observées entre $K_{m}$ de sol différents. Le rôle des constituants du sol a donc, dans la valeur du $K_{m}$ d'un sol, plus d'importance que les fertilisations phosphatées utilisées par l'agriculteur. On peut admettre que le phosphore d'un sol donné possède une constance moyenne de vitesse d'échange des ions phosphate que, ni les prélèvements par les plantes, ni les traitements fertilisants ne sont en mesure de modifier durablement à un coût compatible avec l'économie agricole. Dans la mesure où ces constantes, qui caractérisent les constituants phosphatés du sol, ne sont que peu modifiées par les additions d'engrais phosphatés 
Tableau III. Paramètres des cinétiques d'échanges isotopiques, constantes moyennes de vitesse de transfert et flux de transfert entre la phase solide et la phase liquide $\left(P_{1 F}:\right.$ apport de sous forme de fumier; $P_{1 M}:$ apport de $P$ sous forme minérale).

\begin{tabular}{|c|c|c|c|c|c|c|c|c|c|c|}
\hline \multirow{2}{*}{$\begin{array}{l}\text { Dispositif } \\
\text { et } \\
\text { Traitement }\end{array}$} & & \multirow{2}{*}{$\begin{array}{l}\text { Bilan } \\
F-E \\
k g(P) . h a^{-1}\end{array}$} & \multicolumn{3}{|c|}{ Paramètres des cinétiques } & \multirow[b]{2}{*}{$\begin{array}{l}\mathrm{E}_{\mathrm{Piel}} \\
m g(P) . \digamma^{1}\end{array}$} & \multirow{2}{*}{$\begin{array}{l}E_{P i e} / C p \\
I . \mathrm{kg}^{-1}\end{array}$} & \multicolumn{3}{|c|}{ Constantes moyennes } \\
\hline & & & $\begin{array}{l}{ }^{c_{p}} \\
m g(P) . t^{-1}\end{array}$ & $\mathrm{r} 1 / \mathrm{R}$ & $\mathrm{n}$ & & & $\begin{array}{l}\mathrm{K}_{\mathrm{m}} \\
\left(\min ^{-1}\right)\end{array}$ & $T_{m}$ & 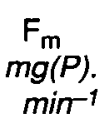 \\
\hline Tennie & 1976 & & 1,2 & 0,51 & 0,14 & 24,5 & 20,4 & 17 & 0,059 & 214 \\
\hline$P_{0}$ & 1988 & -332 & 0,5 & 0,39 & 0,20 & 14,0 & 28 & 22 & 0,045 & 119 \\
\hline$P_{1 F}$ & 1988 & 0 & 0,87 & 0,45 & 0,16 & 18,8 & 21,6 & 23 & 0,043 & 204 \\
\hline$P_{1 M}$ & 1988 & 0 & 0,83 & 0,44 & 0,17 & 18,4 & 22,1 & 21 & 0,047 & 176 \\
\hline$P_{2}$ & 1988 & 332 & 1,2 & 0,52 & 0,13 & 24,0 & 20 & 20 & 0,050 & 248 \\
\hline Herbi & 1981 & & 0,3 & 0,27 & 0,25 & 11,1 & 37 & 47 & 0,021 & 141 \\
\hline$P_{0}$ & 1989 & -250 & 0,13 & 0,15 & 0,29 & 8,7 & 66,9 & 201 & $5.10^{-3}$ & 261 \\
\hline$P_{1}$ & 1989 & 250 & 0,23 & 0,20 & 0,26 & 11,8 & 51,3 & 127 & $8.10^{-3}$ & 291 \\
\hline$P_{2}$ & 1989 & 800 & 0,45 & 0,27 & 0,22 & 17,0 & 37,7 & 85 & 0,012 & 377 \\
\hline \multicolumn{11}{|l|}{$B \times 21$} \\
\hline$P_{0}$ & & -86 & 0,42 & 0,47 & 0,16 & 8,9 & 21,1 & 17,9 & 0,056 & 75 \\
\hline$P_{1}$ & & 30 & 0,59 & 0,49 & 0,16 & 12,5 & 21,1 & 13,8 & 0,072 & 82 \\
\hline$P_{2}$ & & 137 & 0,66 & 0,49 & 0,15 & 13,3 & 20,1 & 17,4 & 0,056 & 116 \\
\hline \multicolumn{11}{|l|}{$B \times 23$} \\
\hline$P_{0}$ & & -137 & 0,22 & 0,16 & 0,27 & 13,0 & 59 & 250 & $4.10^{-3}$ & 562 \\
\hline$P_{1}$ & & 65 & 0,34 & 0,18 & 0,25 & 18,2 & 53,5 & 228 & $4.10^{-3}$ & 784 \\
\hline$P_{2}$ & & 265 & 0,54 & 0,23 & 0,21 & 22,7 & 42 & 180 & $6.10^{-3}$ & 971 \\
\hline$P_{3}$ & & 520 & 0,68 & 0,23 & 0,21 & 28,9 & 42,5 & 204 & $5.10^{-3}$ & 1370 \\
\hline \multicolumn{11}{|l|}{$B \times 24$} \\
\hline$P_{0}$ & & -161 & 0,85 & 0,68 & 0,083 & 12,4 & 14,5 & 4,4 & 0,22 & 37 \\
\hline$P_{1}$ & & 69 & 1,0 & 0,68 & 0,089 & 15,2 & 15,2 & 4,4 & 0,22 & 46 \\
\hline$P_{2}$ & & 332 & 1,4 & 0,69 & 0,073 & 20,3 & 11,0 & 5,1 & 0,20 & 72 \\
\hline \multicolumn{11}{|l|}{$B \times 33$} \\
\hline$P_{0}$ & & -113 & 0,11 & 0,18 & 0,26 & 6,1 & 55,4 & 190 & $5.10^{-3}$ & 213 \\
\hline$P_{1}$ & & 68 & 0,16 & 0,22 & 0,24 & 7,3 & 45,6 & 135 & $7.10^{-3}$ & 211 \\
\hline$P_{2}$ & & 254 & 0,22 & 0,23 & 0,23 & 9,6 & 43,6 & 160 & $6.10^{-3}$ & 347 \\
\hline \multicolumn{11}{|l|}{$B \times 37$} \\
\hline$P_{0}$ & & -110 & 0,29 & 0,21 & 0,21 & 13,5 & 46,5 & 357 & $3.10^{-3}$ & 1024 \\
\hline$P_{1}$ & & 57 & 0,42 & 0,24 & 0,20 & 17,5 & 41,7 & 342 & $3.10^{-3}$ & 1436 \\
\hline$P_{2}$ & & 233 & 0,50 & 0,26 & 0,19 & 19,4 & 38,8 & 311 & $3.10^{-3}$ & 1557 \\
\hline$P_{3}$ & & 484 & 0,94 & 0,29 & 0,16 & 32,0 & 34,0 & 352 & $3.10^{-3}$ & 3302 \\
\hline
\end{tabular}

solubles dans l'eau, on peut conclure que les composés phosphatés qui se forment à l'issue des apports répétés pluri-annuels sont de même nature que ceux préexistant dans le sol. Ce résultat confirme des informations obtenues par extraction chimique séquentielle de différents composés phosphatés des sols (Chang et Jackson, 1957). L'utilisation de cette méthode, pratiquée sur des échantillons de terre prélevés dans des dispositifs de longue durée, avait fait apparaître qu'à l'issue d'apports d'engrais phosphatés solubles dans l'eau, la distribution du phosphore apporté, dans les différents composés phospha- tés du sol, s'effectuait, in fine, à l'image de celle des formes préexistantes.

Ce résultat doit être confronté à ceux observés immédiatement après des additions de phosphate : dans ces dernières circonstances, les constantes de vitesse diffèrent significativement de celles des échantillons de départ (Fardeau, 1981); ce résultat illustre, par la voie des caractéristiques cinétiques, la transformation souvent rapide des composés phosphatés néoformés (Barrow, 1980).

Pour les ions phosphate présents dans des mélanges sol-solution, le paramètre $r_{1} / R$ varie 
de 0,8 à 0,01 et le paramètre $n$ de 0,1 à 0,5 . Les valeurs de $K_{m}$ ont été calculées dans les intervalles indiqués pour $r_{1} / R$ et $n$ (tableau IV). Des valeurs égales de $K_{m}$ sont obtenues pour des couples $r_{1} / R$ et $n$ très différents, c'est-à-dire pour des sols contenant des constituants très différents. Cette observation d'origine théorique pourrait expliquer l'absence quasi générale de relations entre les informations pédologiques et la fertilité phosphorique des sols (Morel et Fardeau, 1987). Alors que rien n'interdit en toute théorie quelque combinaison que ce soit entre $r_{1} / R$ et $n$, seules certaines semblent exister dans les sols portant des plantes : elles apparaissent dans la zone centrale encadrée du tableau IV. Les valeurs situées en haut et à gauche de ce tableau concernent des sols tels que $B \times 24$ où le sable est dominant et les valeurs situées en bas et à droite de ce tableau sont représentatives des sols dont les teneurs en argiles sont supérieures à $40 \%$.

\section{Variations du temps moyen de séjour des ions phosphate dans la solution du sol}

Ce paramètre (tableau III, colonne 9) est l'inverse du précédent; à son image, il est peu influencé, pour un sol donné, par les différents traitements phosphatés. L'information originale est que ce temps moyen de séjour, qui représente le temps nécessaire pour que la quantité d'ions phosphate présente en solution soit renouvelée en totalité par une quantité égale en provenance de la phase solide, est très court; il est inférieur à la minute dans tous les sols analysés pourtant très différents les uns des autres.

Tableau IV. Variations de la constante moyenne de vitesse d'échange, $K_{m}$, en fonction des valeurs des paramètres $\pi / R$ et $n$.

\begin{tabular}{cccccc}
\hline$n$ & 0,1 & 0,2 & 0,3 & 0,4 & 0,5 \\
$r, R$ & & & & & \\
\hline & & & & & \\
0,8 & 0,93 & 0,61 & 0,63 & 0,70 & 0,78 \\
0,6 & 16,5 & 2,6 & 1,6 & 1,4 & 1,4 \\
0,4 & $9,5.10^{2}$ & 18,5 & 6,4 & 3,9 & 3,1 \\
0,2 & $9,7.10^{55}$ & $6,2.10^{2}$ & 64 & 22,3 & 12,5 \\
0,05 & $10^{9}$ & $20.10^{3}$ & $6,4.10^{2}$ & $1,2.10^{2}$ & 50 \\
0,01 & $10^{12}$ & $6,4.10^{8}$ & $6,5.10^{3}$ & $7,1.10^{2}$ & $2.10^{2}$ \\
\hline
\end{tabular}

Variations des flux moyens de transfert des ions phosphate de la phase solide à la phase liquide

Comme les constantes de vitesse et les temps de séjour, les flux moyens diffèrent significativement entre sols; mais à la différence des premiers, ils varient dans une plus grande amplitude pour un sol donné avec les traitements phosphatés. Le résultat le plus inattendu concerne la valeur absolue élevée de ces flux d'ions $\mathrm{PO}_{4}$ entre phase solide et phase liquide qui, dans les échantillons de terre étudiés, n'est jamais inférieure à $30 \mathrm{mg}(\mathrm{P}) \cdot \mathrm{min}^{-1} \cdot \mathrm{kg}(\mathrm{sol})^{-1}$. Dans les meilleures conditions de croissance (climat, caractéristiques physiques du sol, conditions phytosanitaires), $F_{m}$, c'est-à-dire le flux de renouvellement des ions $\mathrm{PO}_{4}$ dans la solution, ne semblerait donc pas pouvoir constituer l'unique facteur limitant l'absorption racinaire du phosphore et par là, le rendement des cultures. La limitation des rendements en relation avec la nutrition phosphatée aurait donc pour origine les conditions régnant dans les sols qui rendraient impossible la réalisation effective de ce flux potentiel.

\section{Variations de rendement des cultures et des valeurs des paramètres $\mathrm{K}_{\mathrm{m}}$ et $\mathrm{F}_{\mathrm{m}}$ en fonction de la fertilisation phosphatée}

Ces relations sont établies en comparant les données du tableau I colonne 10 , et celles du tableau III, colonnes 8 et 10 .

Il a été souligné que les valeurs de $K_{m}$ sont peu affectées par le régime de fertilisation phosphatée; la date de prélèvement des échantillons de terre analysés en est la raison vraisemblable : les engrais phosphatés solubles dans l'eau appliqués ayant suffisamment vieilli au contact du sol, pendant la durée d'un an et plus qui sépare la date de leur application de celle du prélèvement de terre effectué généralement après la récolte. C'est pourquoi, le paramètre $K_{m}$ ne peut permettre de rendre compte des variations possibles du rendement des cultures avec la fertilisation phosphatée. Cependant, les modifications de $K_{m}$ observables sur des échantillons de terre prélevés très peu de temps après l'incorporation des engrais phosphatés dans le sol pourraient expliquer les effets immédiats des apports de phosphore sur la production végétale. Quant aux flux d'échange, $F_{m}$, c'est dans les sols où ils sont les plus faibles ( $B \times 21, B \times 24, \ldots$ ) que sont observés des accroissements de rendements des cultures par la fertilisation phosphatée. 
Le facteur flux solide $\rightarrow$ liquide paraît donc pouvoir contrôler la nutrition phosphatée des cultures.

L'exemple de l'essai $B \times 24$ dont les résultats sont commentés ci-après illustre bien cette observation. Le sol sableux de cet essai contient des quantités importantes de phosphore potentiellement assimilable tant à l'analyse chimique (Dyer) qu'à l'analyse isotopique (E1) même après 6 ans sans apport de phosphore ( $P$ Dyer $=130$ $\mathrm{mg}(P) \cdot \mathrm{kg}^{-1} ; \mathrm{E1}=12,4 \mathrm{mg}(P) \cdot \mathrm{kg}^{-1}$ ); le facteur intensité, $c p$, dépasse largement la valeur de 0,2 $\mathrm{mg}(\mathrm{P}) . \mathrm{I}^{-1}$ considérée comme nécessaire et suffisante pour assurer une alimentation satisfaisante des cultures (Barber, 1984). Néanmoins, une augmentation régulièrement significative des rendements du maïs cultivé sous irrigation dans ce sol profond est obtenue avec des apports annuels d'engrais phosphatés soluble-eau, la dose appliquée la plus élevée $\left(87 \mathrm{~kg}(\mathrm{P}) \cdot \mathrm{ha}^{-1}\right)$ ne se montrant que parfois supérieure à celle de $40 \mathrm{~kg}$ $(P) \cdot \mathrm{ha}^{-1}$. Les caractéristiques chimiques et isotopiques d'une part et agronomiques d'autre part sont donc contradictoires. Cependant, les flux de transferts potentiels sont les plus faibles de ceux mesurés sur les échantillons étudiés, particulièrement pour $P o$. Mais d'après la valeur absolue de ce flux pour $P o$, ce flux pourrait encore suffire à satisfaire les exigences en $P$ du maïs.

C'est un fait établi que toute valeur issue d'une analyse chimique ou isotopique d'une terre où toute la terre participe aux échanges entre sol et solution est une valeur potentielle. Dans la réalité du champ, seulement $1-2 \%$ du sol au maximum est en contact, à un instant donné, avec le système racinaire actif et la plante ne peut donc bénéficier dans le meilleur des cas et en permanence que de $1-2 \%$ des possibilités de renouvellement des ions de la phase liquide par ceux de la phase solide. C'est pour cette raison, et aussi parce que les ions phosphate migrent peu, que le flux de transfert des ions phosphate devient le facteur limitant de la nutrition phosphatée.

Cette approche des mécanismes de nutrition phosphatée dans les sols en place permet de comprendre pourquoi, dans un sol donné, d'une part des plantes ou des variétés différentes pourront réagir ou non à des apports d'engrais phosphaté en raison des différences dans leur système racinaire, et d'autre part lorsque le flux potentiel est limité, une plante donnée, peut ou non réagir à des apports en fonction de l'état de son système racinaire. Quant aux valeurs seuils des flux qui permettraient de prévoir avec certitude l'aptitude réelle d'un sol donné à assurer de manière satisfaisante la nutrition phosphatée d'une succession de culture - en l'absence de facteurs autres que $P$ limitant la croissance -, fera l'objet d'un travail ultérieur. Mais on peut dès maintenant indiquer que la valeur de $80 \mathrm{mg}$ (P) $\mathrm{min}^{-1}$. (kg.sol) $)^{-1}$ pourrait être ce seuil pour les plantes de grande culture dont les rendements seraient proches du rendement potentiel.

\section{CONCLUSION}

La nutrition phosphatée des végétaux dans les sols en place ne peut être expliquée que si les ions phosphate de la solution du sol sont renouvelés 10 à 100 fois plus vite que la solution du sol dont l'eau est consommée pour assurer la transpiration. C'est donc la vitesse de renouvellement qui paraît être le facteur primordial de cette nutrition alors que l'on n'évalue que des quantités de phosphore éventuellement assimilable par utilisation des méthodes conventionnelles d'analyse de $P$ du sol. En faisant appel aux techniques des cinétiques d'échanges isotopiques, il a été possible de déterminer des vitesses et des flux de renouvellement des ions phosphate de la solution du sol par des ions en provenance de la phase solide. La comparaison des données relatives aux flux de renouvellement et aux rendements des cultures permet de mettre en évidence, comme on le pressentait, le rôle déterminant des flux dans la nutrition phosphatée et, par là, dans la réaction des cultures aux apports d'engrais frais. D'après les résultats obtenus sur les échantillons de terre étudiés, les flux d'ions phosphate entre phases solide et liquide seraient amplement suffisants si les racines avaient la possibilité d'exploiter la totalité du sol. Mais on sait que l'exploitation racinaire ne concerne qu'une très faible fraction des surfaces des particules, tout en pouvant être très variable d'une plante à l'autre en fonction des conditions climatiques ou de structure du sol et d'une espèce végétale à l'autre. Cette analyse permet d'expliquer pourquoi les rendements d'une culture donnée sur un sol donné peuvent, en fonction des années, être ou non modifiés par un apport récent d'engrais.

Cette approche des mécanismes de la nutrition phosphatée conduit à privilégier l'alimentation hydrique pour assurer la nutrition phosphatée. On sait depuis longtemps que le rendement 
d'une plante comme le riz inondé n'est pratiquement jamais modifié par des apports d'engrais phosphatés même lorsque les sols ont des teneurs en phosphore assimilable très faibles alors que les rendements des cultures pluviales dans les mêmes sols peuvent être accrus par des apports d'engrais. En culture inondée ou humide, le renouvellement des ions phosphate de la solution du sol est assuré en permanence, ce qui ne peut être le cas en culture sèche.

La nutrition phosphatée des plantes ne peut être assurée de manière continue que si un renouvellement, en quantité suffisamment importante, du phosphore de la solution du sol peut être assuré. Ce fait a 2 conséquences agronomiques essentielles :

- la présence d'eau entre la phase solide du sol et la racine est indispensable, même lorsque la plante dispose, pour son évapotranspiration, d'une quantité d'eau juste suffisante;

- le renouvellement local des ions phosphate pourra être d'autant plus faible pour assurer le prélèvement par une plante d'une quantité donnée que le système racinaire actif sera plus développé.

Les résultats concernant les flux d'ions phosphate et les hypothèses relatives à l'alimentation hydrique racinaire concomitante devraient guider les futures recherches en matière de raisonnement de la fertilisation phosphatée.

\section{RÉFÉRENCES}

Barber SA (1984) Soil nutrient bioavailability. A mechanistic approach. John Wiley. New York

Barrow NJ (1980) Evaluation and utilization of residual phosphorus in soils. In: The role of phosphorus in agriculture (Khasawneh FE, Sample EC, Kamprath EJ, eds), Am Soc Agron Madison, WI 333-359

Boniface R, Trocme S (1988) Enseignements fournis par des essais de longue durée sur la fumure phosphatée et potassique. 2. Essais sur la fumure phosphatée. In: Phosphore et Potassium dans les relations sol-plante : conséquence sur la fertilisation ( $\mathrm{L}$ Gachon, ed) INRA, Paris, 279-402

Calvet R (1988) Analyse du concept de biodisponibilité d'une subtance dans le sol. Sci Sol 2, 183-202

Chang SC, Jackson ML (1957) Fractionation of soil phosphorus. Soil Sci 84, 133-144

Conesa AP (1967) Emploi d'une résine anionique pour l'étude du "potentiel phosphate" des sols. In: Colloque Int phosphates Minér solides. Vol 2 :
Phosphates naturels. Phosphates dans l'agriculture. CNRS Paris, 125-129

Coulter BS (1969) Calculation of precision in isotope dilution experiments. Int $J$ App/ Radiat Isot 20, 271 . 274

Crank J (1975) The mathematics of diffusion. Clarendon Press, Oxford

Daubeny $D$ (1845) On the distinction between the dormant and active ingredients of the soil. JR Agric Soc Engl 7, 1, 237-245

Fardeau JC (1981) Cinétiques de dilution isotopique et phosphore assimilable des sols. Thèse Univ Paris VI, $198 p$

Fardeau JC, Jappe J (1988) Valeurs caractéristiques des cinétiques de dilution isotopique des ions phosphate dans les systèmes sol-solution. In: Phosphore et potassium dans les relations sol-plante: conséquence sur la fertilisation ( $\mathrm{L}$ Gachon, ed) INRA, Paris, 78-99

Fardeau JC, Morel C, Jappe J (1985) Cinétique d'échange des ions phosphate dans les systèmes sol-solution. Vérification expérimentale de l'équation théorique. CR Séances Acad Sci Paris t 300 , Sér III, 8, 371-376

Feller W (1966) An introduction to probability theory and its applications. Vol II. John Wiley, New York

Gachon L (1977) Utilité d'un bon niveau de réserves phosphatées du sol. Phosphores Agric 70, 27-33

Gachon L (1988) Introduction. In: Phosphore et potassium dans les relations sol-plante : conséquence sur la fertilisation (L Gachon, ed) INRA, Paris, 7-10

John MK (1968) Colorimetric determination of phosphorus in soil and plant materials with ascorbic acid. Soil Sci 109, 4, 214-220

Larsen $S$ (1967) Isoionic exchange of phosphate in paddy soils. Plant Soil 27, 401-407

Mariotti A (1982) Apports de la géochimie isotopique à la connaissance du cycle de l'azote. Thèse Univ Paris VI, $660 \mathrm{p}$

Morel C (1988) Analyse par traçage isotopique, du comportement du phosphore dans les systèmes sol-engrais-plante : conséquence en matière de fertilisation. Thèse Univ Aix-Marseille III, $165 p$

Morel C, Fardeau JC (1987) Le phosphore assimilable des sols intertropicaux : ses relations avec le phosphore extrait par deux méthodes chimiques. Agron Trop 42, 4, 248-257

Morel C, Fardeau JC (1990) Phosphorus bioavailability of fertilizers : a predictive laboratory method for its evaluation. Fertil Res 28, 1-9

Roche P (1983) Les méthodes d'appréciation du statut phosphorique des sols. Leur application à l'estimation des besoins en engrais phosphatés. In: Actes du $3 e$ congr int composés phosphorés (Imphos, ed) Casablanca, Maroc, 165-194

Sen Tran T, Fardeau JC, Giroux M (1988) Effects of soil properties on plant-available phosphorus deter- 
mined by the isotopic dilution phosphorus-32 method. Soil Sci Soc Am J 52, 5, 1383-1390

Sheppard CW (1962) Basic principles of the tracer method. Introduction ot mathematical tracer kinetics. John Wiley, New York

Shipley A, Clark RE (1972) Tracer methods for in vivo kinetics. Theory and applications. Acad Press, New York

Sibbessen $E$ (1978) An investigation on the anion exchange resin method for soil phosphate extraction. Plant Soil 50, 305-321

Taureau JC, Courant G (1988) Essai de Tennie (72). Perspect Agric 127, 228-233
Taureau JC, Bourtembourg J, Savary C (1988) Essai d'Herbisse (10). Perspect Agric 127, 193-203

Triboï E, Gachon L (1988) Étude des méthodes d'appréciation de l'offre phosphatée du sol. 1. Détermination du phosphore isotopiquement échangeable ou valeur E. In: Phosphore et potassium dans les relations sol-plante : conséquence sur la fertilisation (L Gachon, ed) INRA, Paris, 165-180

Wiklander $L$ (1950) Kinetics of phosphate exchange in soils. Ann R Coll Agric Swed 17, 407-424

White RE, Beckett PHT (1964) Studies on the phosphate potentials of soils. Part I. The measurement of phosphate potential. Plant Soil 20, 1, 1-16 